The amount of amylose required per unit volume of juice (for example, 13 per cent $w / v$ above) depends on the previous history of the amylose, and particularly on the extent to which it has retrograded during preparation. The pea amylose used was a rather insoluble alcohol-dried sample. Potato amylose and potato whole starch also adsorb the enzymes under the conditions described; but the use of whole starch is not advocated owing to the complications introduced by its content of amylopectin. A non-retrograded, undried sample of potato amylose was found to adsorb 50 per cent of the phosphorylase of the potato juice when added to the extent of only 3 per cent $\mathrm{w} / \mathrm{v}$ of the juice. Moreover, the whole of the $Q$-enzyme was adsorbed by 0.15 per cent of this amylose, enabling the $Q$-enzyme to be removed from the phosphorylase at this stage if desired.

Part of the adsorbed $Q$-enzyme is firmly bound to the amylose and cannot be removed with salt solution. It can only be removed by washing the precipitate with starch solution. This suggests that there is a general attraction of the amylose chains for the $Q$-enzyme molecule (presumably through secondary valency forces) leading to a complex which is easily dissociated in salt solution, and that in addition there are a number of sites at which the full enzymesubstrate complex is formed. From these sites the enzyme can be removed only by presenting the substrate in the dissolved form.

It will be noticed that the method of preparation above resembles that used by Schwimmer and Balls ${ }^{6}$, who obtained $\alpha$-amylase from barley by adsorption of the amylase on starch grains from alcoholic solution, and by Hobson, Whelan and Peat ${ }^{7}$, who removed $Q$-enzyme irreversibly on potato starch in the preparation of $R$-enzyme.

We wish to thank Prof. H. W. Melville and Prof. M. Stacey for their interest in this work. One of us (H. B.) is indebted to the Department of Scientific and Industrial Research for a maintenance grant.

\section{H. BAUM}

G. A. Gilbert

Department of Chemistry, University, Edgbaston,

Birmingham 15. Nov. 18.

${ }^{2}$ Hanes, C. S., Proc. Roy. Soc., B, 128, 421 (1940); B, 129, 174 (1940). ${ }^{2}$ Gilbert, G. A., and Patrick, A. D., Biochem. J., 51, 186 (1952).

${ }^{3}$ Haworth, W. N., Peat, S., and Bourne, E. J., Nature, 154, 236 (1944).

" Gilbert, G. A., and Patrick, A. D., Biochem. J., 51, 181 (1952).

'Hilbert, G. E., and MacMasters, M. M., J. Biol. Chem., 162, 229 (1946).

- Schwirmer, S., and Balls, A. K., J. Biol. Chem., 179, 1063 (1949).

'Hobson, P. N., Whelan, W. J., and Peat, S., Biochem. J., 47, xxxix (1950); J." Chem. Soc., 1451 (1951).

\section{A Method of Embedding Brittle Specimens of Wood for Sectioning}

GREAT difficulty was encountered in cutting sections from the brittle and often partly carbonized wooden remains found on the sites of Roman and ancient British settlements. The impact of the razor caused instantaneous fragmentation of the sections, and identification became very difficult. A simple embedding material was sought which would penetrate the specimen without difficulty, and which could be cut easily and would prevent the resulting section from crumbling.

A proprietary brand of a polyvinyl lactophenol mixture (that is, polyvinyl alcohol in lactic acid and phenol) was used at first, as it was known that this substance had proved satisfactory in the mounting of entomological specimens without the necessity of previous dehydration. The procedure adopted was as follows. Cubes of the brittle wood are cut in the usual way, with a tangential, a radial and a transverse face ; they are then immersed in the polyvinyl lactophenol mixture and warmed gently for half an hour. After removal from the liquid, the cubes were allowed to drain and cool for $24 \mathrm{hr}$. (It will be found that the cubes are now darker in colour and of a rubbery consistency.) The cubes can then be sectioned with a sharp razor, and the sections mounted directly on to a slide, using the embedding agent as a mounting medium.

The resulting preparation possesses the added advantage of being 'cleared' by the polyvinyl lactophenol. The cell walls, which may be blackened and beginning to undergo carbonization, are rendered clear and translucent, enabling details of structure to be easily observed.

The method has proved to be a quick, easy and efficient one, but possesses certain aspects where care has to be taken. Due to the rubbery nature of the material, sections have to be cut with a sharp razor or else the cube will bend under the impact of the blade. Staining is somewhat difficult, because many dyes tend to diffuse from the sections into the mounting medium'. It has proved possible to stick the section to a slide, wash off the polyvinyl lactophenol in hot water, and then to stain, clear and mount up in canada balsam in the usual way, but this technique still requires to be perfected. Some preparations of polyvinyl lactophenol (for example, the proprietary one already mentioned) cause a slight shrinkage to the tissues on drying. Other preparations dry more slowly and so require a longer period at the second and third stages outlined above (for example, Salmon's type $M . A .1^{2}$ ).

Subsequent search through the literature has shown that this method is not new. Lubkin and Carsten ${ }^{3}$ have used a polyvinyl alcohol-glycerine mixture for embedding and sectioning medical pathological material, such as heart, lungs, liver and spleen. Gray and Wess ${ }^{4}$ advocate a polyvinyl alcoholglycerine - lactic acid - acetone mixture for mounting entomological specimens, which has a slightly lower refractive index, but which Brown ${ }^{5}$ claims overcomes the tendency to shrink and sets harder than other preparations. 'These preparations and others are being tested and may well eliminate some of the difficulties in the technique mentioned. Even with these disadvantages, the ease and quickness of embedding and mounting the material should commend itself as a useful method, especially where speed in identification of specimens is an important consideration.

\section{J. F. LEVY}

Botany Department,

Imperial College of Science and Technology, London, S.W.7. Dec. 12.

1 Vetcaife, C. R., and Richardson, F. R., Lew Bull., 569 (1950).

'Salmon, J. T., The Microscope, 8, 139 (1951).

${ }^{3}$ Jubkin, V., and Carsten, M., Science, 95, 633 (1942).

${ }^{4}$ Gray, P., and Wess, G., J. Roy. Micro. Soc., Y0, 287 (1950).

- Brown, H. J., The Microscope, 8, 263 (1951). 